\title{
Driven reconnection and bursty bulk flows
}

\author{
B. P. Pandey ${ }^{1}$ and G. S. Lakhina ${ }^{2}$ \\ ${ }^{1}$ Centre for Plasma Astrophysics, K. U. Leuven, Celestijnenlaan 200B, 3001 Heverlee, Belgium \\ ${ }^{2}$ Indian Institute of Geomagnetism, Bombay 400 005, India
}

Received: 29 May 2000 - Revised: 2 April 2001 - Accepted: 11 May 2001

\begin{abstract}
The energetics of driven magnetic reconnections induced by the deformation of the magnetopause boundary due to the solar wind-magnetosphere interaction are studied. The bursty type reconnection ensues due to the forcing of the magnetopause boundary by the solar wind. For typical plasma parameters in the inner central plasma sheet (ICPS), the magnetic energy release during the reconnection is estimated and it is found that the available free energy is comparable to the observed kinetic energy of typical bursty bulk flows. It implies that the part of the free energy goes into the heating of the ICPS particles, whereas the rest goes into its acceleration. The accelarated particle manifests itself as bursty flows.
\end{abstract}

Key words. Magnetospheric physics (magnetotail; storms and substorms)

\section{Introduction}

Observations of the near-Earth plasma sheet between approxiamately 10 and $20 R_{E}$ suggest that the main transport of mass, energy and magnetic flux is realized via shortlived, high speed plasma flows, known as bursty bulk flows (BBFs) (Angelopolous et al., 1992, 1994, 1997; Baumjohann and Paschmann, 1990; Baumjohann, 1993; Sergeev et al., 1995; Yermolaev et al., 1999). The occurrence rates of high speed flows in the plasma sheet boundary layer (PSBL) and inner central plasma sheet (ICPS) are in the ratio of $2: 1$ for 400 $600 \mathrm{~km} / \mathrm{s}$ flows and 1:2 for flows greater than $800 \mathrm{~km} / \mathrm{s}$. The flow bursts are directed predominantly Earthward and they are associated with relatively low plasma density in all plasma sheet regions. A typical BBF event lasts for about 10-15 minutes and a majority of all flows stay uninterupted at high speed levels for around $10 \mathrm{~s}$.

The transport of plasma and magnetic fluxes at a high speed, both Earthward and tailward, have been explained in

Correspondence to: B. P. Pandey

(birendra.pandey@wis.kuleuven.ac.be) the framework of the near-Earth neutral line (NENL) model (Hones, 1976; Hones and Schindler, 1979; Birn, 1980). The NENL model has been quite successful in explaining many diverse observed features of substorm related changes in the magnetotail. Even though BBFs are positively correlated with the geomagnetic activity, there is no one-to-one correspondence between a substorm and BBF. Therefore, the NENL paradigm cannot be invoked to explain the bursty flows. There have been various models proposed so far for the bursty bulk flows. Russel et al. (1994) suggested that BBFs can be triggered by the sudden changes in solar wind dynamic pressure. Lakhina $(1992,1996)$ proposed a mechanism for BBFs based on the bursty driven reconnection in the magnetotail. In contrast to the spontaneous reconnection which occurs as a result of the resistive MHD instabilities (such as tearing mode, kink mode etc.), driven or forced reconnection is produced by external deformation of the initially smooth magnetic field through perturbation at the boundaries even when magnetic equilibrium is stable against the resistive mode.

Recently, the formation of a thin current sheet in the presence of an electric field at the boundary (which mimics the solar wind-magnetospheric interaction) has been investigated by Birn et al. (1998). The problem of driven reconnection has been investigated by several authors (Vekstein and Jain, 1998, 1999; Ishizawa and Tokuda, 2000; Browning et al., 2001). The energetic aspect of driven reconnection has been investigated analytically in the context of solar coronal heating by Vekstein and Jain $(1998,1999)$ and numerically by Browning et al. (2001). These studies conclude that the heating of the current sheet is most efficient when the ongoing external driving frequency is of the order of a tearing time scale. The numerical study of Browning et al. (2001) suggests that a small deformation of the boundary can cause sufficient release of magnetic energy in the plasma sheet. In the present work, we shall follow the approach of Vekstein and Jain (1998) and investigate the energetic aspect of driven magnetic reconnection and its implication for plasma heating in a two-dimensional current sheet (Birn et al., 1975) which ap- 


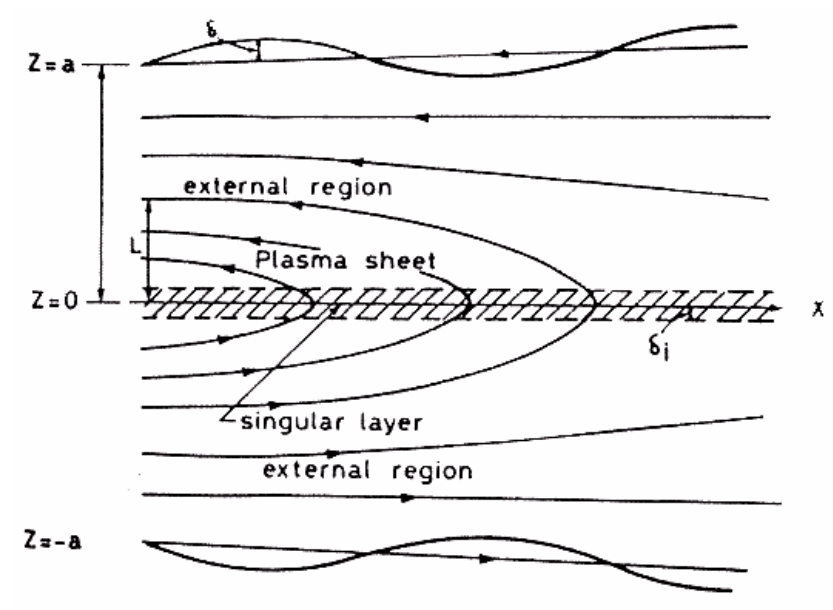

Fig. 1. Schematics of the Earth's magnetotail: The magnetotail has a characteristic dimension of $2 L$ in the $z$-direction. The magnetopause is perturbed by $\delta$ due to its interaction with the solar wind. The $z=0$ is the layer of singularity and has a width of twice the ion-Larmor radius.

proximates the Earth's magnetotail configuration fairly well. The initial equilibrium state is perturbed by the solar wind at the magnetopause boundary originally located at $z= \pm a$. We shall assume that the perturbation is slow compared with the Alfvén time scale and therefore, the subsequent evolution of the system is quasistatic in the "outer region", i.e. the region external to the "inner region" where dissipative effects are important.

\section{The model}

We consider a two-dimensional slab equilibrium for the Earth's magnetotail, as shown in Fig. 1 (Lakhina, 1992). The field line lies in the $x-z$ plane with the $x$-axis along the tail and pointing away from the Earth, and the $z$-axis, which is parallel to the dipole axis, is assumed perpendicular to the Sun-Earth line. Dawn-dusk variation along $y$ is ignored. The width of the plasma sheet is $2 L$ and the magnetopause boundary is situated at $z= \pm a$. The disturbance in the solar wind will deform the magnetopause boundary

$z_{b}^{ \pm}= \pm[a-\delta(t) \cos (k x)], \quad \delta \ll a$.

Then the deformed flux function can be represented as

$A(x, z, t)=A_{0}(x, z)+A_{1}(x, z, t)$,

where $A_{0}$ and $A_{1}$ refer to the equilibrium and perturbed quantities respectively. The self-consistent equilibrium solution $A_{0}(x, z)$ is obtained by solving the Grad-Shafranov equation (Schindler, 1972; Birn, 1975) by assuming the slow variation of magnetotail quantities along $x$ in comparison with the strong variation along $z$ and assuming that pressure $p\left(A_{0}\right)$ has an exponential profile. Then

$A_{0}(x, z)=-B_{0} L \ln [\cosh (z / L)]+B_{0} L \ln \left[p_{0} / p_{b}\right]$, where $p_{0}(x)=p\left(A_{0}(x, 0)\right)$ is the pressure on the $x$-axis and $p_{b}=p\left(A_{b}\right)$ is the pressure at the magnetopause boundary. We shall assume that the deformed boundary is a magnetic surface and thus, the flux function is constant there and hence, the perturbed flux function $A_{1}$ must be of the form

$A_{1}(x, z, t)=A_{1}(z, t) \cos (k x)$.

The deformation of magnetopause boundary takes place over a few hours and thus, the external driving time scale of the boundary will be very large in comparison with the Alfven time scale which, in this case, is of the order of a few seconds. As a result, the boundary deformation $\delta(t)$ is setup slowly compared to the Alfvén time scale. Then the evolution of $A_{1}(z, t)$ is quasistatic and can be described by following Grad-Shafranov equation (Lakhina and Schindler, 1988; Lakhina, 1992)

$\left[\frac{d^{2}}{d \bar{z}^{2}}-\bar{k}^{2}+\frac{2}{\cosh ^{2} \bar{z}}\right] A_{1}(z, t)=0$,

where $\bar{z}=z / L$ and $\bar{k}=k L$. The boundary condition at the deformed boundary is given by

$A_{1}(a)=-B_{0} \delta \tanh (\bar{a})$,

where $\bar{a}=a / L$.

Equation (5) describes two possible equilibria, which are consistent with the boundary condition (6) (Lakhina, 1992). The first equilibrium

$A_{1}^{i}(z, t)=-\frac{B_{0} \delta \tanh (\bar{a})}{\Phi_{1}(\bar{k}, \bar{a}) \sinh (k a)} \Phi_{1}(\bar{k}, \bar{z}) \sinh (k z)$,

has the property that no flux crosses the $z=0$ plane. The second equilibrium

$A_{1}^{r}(z, t)=-\frac{B_{0} \delta \tanh (\bar{a})}{\Phi_{2}(\bar{k}, \bar{a}) \cosh (k a)} \Phi_{2}(\bar{k}, \bar{z}) \cosh (k z)$,

has a different topology possessing islands of width $w \approx$ $-2 a \tanh (\bar{a}) \delta \bar{k} /\left[\Phi_{2}(\bar{k}, \bar{a}) \cosh (k a)\right]$. Here,

$\Phi_{1}(\bar{k}, \bar{z})=-\bar{k}+\tanh (\bar{z}) / \tanh (\overline{k z})$,

$\Phi_{2}(\bar{k}, \bar{z})=-\bar{k}+\tanh (\bar{z}) \tanh (\overline{k z})$.

Having these two solutions $A_{1}^{i}$ and $A_{1}^{r}$, the transition from the ideal equilibrium $A_{1}^{i}$ to the reconnected equilibrium $A_{1}^{r}$ occurs in several stages (Hahm and Kulsrud, 1985). Initially, after the deformation of the magnetopause, the system approaches the ideal equilibrium $A_{1}^{i}$ by developing a surface current at the resonant surface $z=0$, where the current density increases with time and thickness of the sheet decreases. After sufficient thinning of the sheet, collisionless damping becomes important and the transition to $A_{1}^{r}$ takes place over a $\tau \sim 1 /|\gamma|$ time scale. The expression for $\gamma$ is obtained by matching the singular layer solution of Eq. (5) (i.e. $z<\delta_{i}$ solution) with the solution in the external region $\left(z>\delta_{i}\right)$ at $z=\delta_{i}$ (Lakhina, 1992). Here, $\delta_{i}=\sqrt{a_{i} L / 2}$ and $a_{i}=v_{t i} / \omega_{c i}$ is the ion Larmor radius and 
$v_{t i}=\sqrt{2 T_{i} / m_{i}}, \omega_{c i}=\left(e B_{0} / m_{i} c\right)$ are the thermal speed and gyrofrequency of the ions, $T_{i}$ is the ion temperature and $\gamma$ is given by

$$
\begin{array}{r}
\gamma=g_{0}\left\{\left(1-\bar{k}^{2}\right) Q-\frac{\bar{k}^{3} \sqrt{\overline{a_{i}}}}{\epsilon^{2}\left(1+T_{e} / T_{i}\right)}[(1-\eta)\right. \\
\left.\left.+\frac{64 \eta}{105}\left(\frac{1}{\epsilon \bar{k}}\right)^{3 / 2}\right]\right\},
\end{array}
$$

$g_{0}=\sqrt{\frac{2}{\pi}} \frac{\omega_{c i}{\overline{a_{i}}}^{5 / 2}\left(1+T_{e} / T_{i}\right)}{\left(1+2{\overline{a_{i}}}^{2}\right)}$

$$
Q=\frac{\Phi_{2}(\bar{k}, \bar{a})}{\Phi_{1}(\bar{k}, \bar{a}) \tanh (k a)} \text {. }
$$

Here, $\epsilon=B_{z} / B_{0}$ is a small parameter and $\eta$ is the fraction of trapped electrons in the singular layer. The inductive electric field generated during reconnection causes the plasma flow along the tail axis given by,

$v_{x}=\alpha_{0} \frac{\left(1-\bar{k}^{2}\right) Q g_{0} A_{1}^{r}(z=0) \exp (\gamma t)}{\epsilon B}$

where

$$
\begin{aligned}
\alpha_{0}= & -\frac{-\Phi_{2}(\bar{k}, \bar{z}) \cosh (k z)}{\bar{k}} \\
& +\frac{\Phi_{2}(\bar{k}, \bar{a}) \Phi_{1}(\bar{k}, \bar{z}) \sinh (k z)}{\Phi_{1}(\bar{k}, \bar{a}) \tanh (k a)} \\
& -\frac{B_{0} \delta \tanh (\bar{a}) \sinh (k z)}{\Phi_{1}(\bar{k}, \bar{a}) \sinh (k a)}
\end{aligned}
$$

where $\Phi_{1,2}$ is given by Eqs. (9) and (10). For $\gamma<0$, the driven reconnection lasts for a very brief period and hence it is termed as a bursty type of reconnection. For the parameters of the near-Earth plasma, $|\gamma|$ is typically of the order of one minute or so. The numerical simulation of driven reconnection by Birn et al. (1998) suggests that the maximum amplitude of velocity perturbation is large for a short time scale, which is in conformity with Eq. (14). For typical plasma parameters, e.g. magnetic field $B \sim 25 \mathrm{nT}, a \sim 30 R_{E}$, $\delta_{i} \sim 10^{4} \mathrm{~km}$ during a BBF, one can show (Lakhina, 1996) that the Earthward flow with a bulk velocity $v_{x}>400 \mathrm{~km} \mathrm{~s}^{-1}$ lasting for a few seconds and bulk flows with speeds of $\sim$ $100 \mathrm{~km} \mathrm{~s}^{-1}$ lasting for about one minute or so can be produced by the bursty driven reconnections.

Initially, the energy is stored in the current sheet in the form of magnetic energy and as the reconnection sets in, this energy is released and plasma is heated and accelerated. Therefore, an important characteristic of the transition from one equilibrium state to another, $A_{1}^{i}$ to $A_{1}^{r}$, from the point of view of plasma heating and acceleration, is the amount of magnetic energy released as this energy becomes freely available. This energy can be estimated as the difference in the magnetic energy of the equilibrium, namely $A_{1}^{i}$ and $A_{1}^{r}$. In order to calculate these energies, $W_{B}^{i}$ and $W_{B}^{r}$, we consider the energy balance. Energy of the initial magnetic field $W_{B}^{0}$ (related to the unit area of the slab in the $x-y$ plane) is

$$
\begin{aligned}
W_{B}^{0} & =\int \frac{B_{0}^{2}}{2 \mu_{0}} d z \simeq \int \frac{B_{0 x}^{2}}{2 \mu_{0}} d z \\
& \approx \frac{B_{0}^{2} a}{\mu_{0}}\left[1-\left(\frac{2 L}{a}\right) \tanh (a / L)\right] .
\end{aligned}
$$

The total energy is $W_{B}=W_{B}^{0}+W_{B}^{(i, r)}$, where $W_{B}^{(i, r)}$ is the work done by the external force (solar wind) in deforming the magnetopause boundary which results in the perturbed equilibrium $A_{1}^{(i, r)}$. The force exerted by the solar wind has to balance the magnetic pressure at the boundary surface, $B^{2} /\left(2 \mu_{0}\right)$. Therefore, the work done $W$ is

$$
W=\int_{0}^{t}\left\langle\frac{B\left(z_{b}^{ \pm}\right)^{2}}{2 \mu_{0}} \frac{d \delta}{d t} \cos (k x)\right\rangle d t,
$$

where $\delta(t=0)=0$ is assumed and the angular bracket inside the integrand means an averaging over the periodic variation along $x$-axis. As the deformation of the boundary $\delta$ sets up slowly compared with the Alfvén time scale $\tau_{A}$ $\left(\tau_{A}=\left(a / V_{A}\right), V_{A}=B_{0} / \sqrt{2 \mu_{0} \rho}\right)$, the plasma will be in a magnetostatic equilibrium at any given moment. Therefore, in deriving $W$ in Eq. (17), it is possible to calculate magnetic pressure $B\left(z_{b}^{ \pm}\right)^{2} /\left(2 \mu_{0}\right)$ using $A_{1}^{(i, r)}$. The deformed magnetic field at the boundary $\boldsymbol{B}=\boldsymbol{B}_{i}+\boldsymbol{b}$, where $\boldsymbol{B}_{i}$ is the initial field and $\boldsymbol{b}$ is the perturbed field, is given as

$b_{x}=-\frac{\partial A_{1}}{\partial z}=-A_{1}^{\prime}(z, t) \cos (k x)$,

$b_{z}=\frac{\partial A_{1}}{\partial x}=-k A_{1}(z, t) \sin (k x)$

Thus, the required magnetic pressure at the boundary is

$$
\begin{aligned}
\frac{B^{2}\left(z_{b}^{ \pm}\right)}{2 \mu_{0}}=\frac{B_{i}^{2}}{2 \mu_{0}}+\frac{1}{\mu_{0}}[ & -B_{x 0}(a) A_{1}^{\prime}(a, t) \cos (k x) \\
& \left.-k B_{z 0}(a) A_{1}(a, t) \sin (k x)\right] .
\end{aligned}
$$

Only the term proportional to $\cos (k x)$ makes a non-zero contribution in Eq. (17)

$\left\langle\frac{B\left(z_{b}^{ \pm}\right)^{2}}{2 \mu_{0}} \frac{d \delta}{d t} \cos (k x)\right\rangle=-\frac{B_{x 0}(a)^{2}}{2 \mu_{0}} A_{1}^{\prime} \frac{d \delta}{d t}$.

Therefore,

$W_{B}^{i}=\frac{B_{0}^{2}}{2 \mu_{0}} \frac{\delta^{2}}{2 L} F(k a)$,

and

$W_{B}^{r}=\frac{B_{0}^{2}}{2 \mu_{0}} \frac{\delta^{2}}{2 L} G(k a)$, 


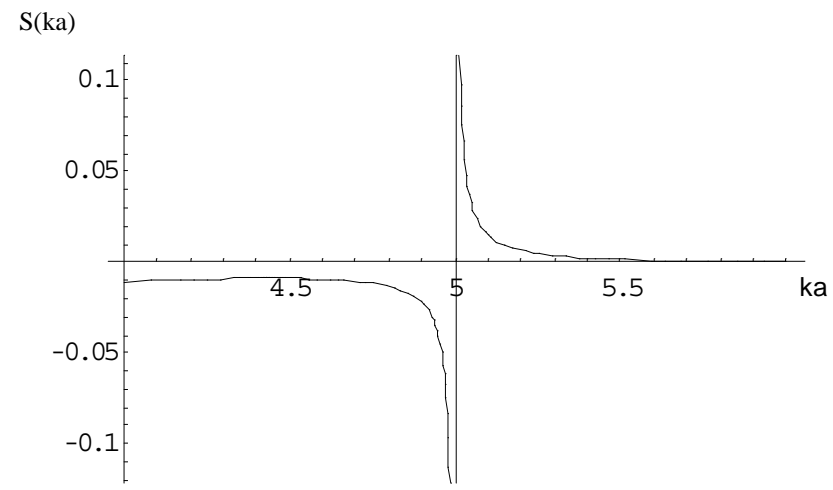

Fig. 2. The factor $S(k a)$ plotted as a function of $k a$ for $L \sim 6 R_{E}$, $a \sim 30 R_{E}$ with $\left|B_{z}\right| \sim 25 \mathrm{nT}$ and $\delta_{i} \sim 10^{4} \mathrm{~km}$. We see from the figure that for short scale length $k a \geq 5$ this function is positive.

where

$$
\begin{aligned}
& F(k a)=\tanh (a / L) \\
& \times\left[\frac{\cosh ^{-2}(a / L) / \tanh ^{2}(k a)-k L \tanh (a / L) / \sinh ^{2}(k a)}{-k L+\tanh (a / L) / \tanh (k a)}\right. \\
& \left.+\frac{k L}{\tanh (k a)},\right]
\end{aligned}
$$

and

$$
\begin{array}{r}
G(k a)=\tanh (a / L) \\
\times\left[\frac{\cosh ^{-2}(a / L) \tanh (k a)+k L \tanh (a / L) \cosh ^{-2}(k a)}{-k L+\tanh (a / L) \tanh (k a)}\right. \\
\left.+\frac{k L}{\tanh (k a)} .\right]
\end{array}
$$

Next, we can calculate the energy released per unit volume $\Delta W=\left(W^{i}-W^{r}\right) / 2 \delta_{i}$ which is

$\Delta W=\frac{B_{0}^{2}}{2 \mu_{0}}\left(\frac{\delta}{\delta_{i}}\right)^{2} S(k a)$,

where $S(k a)=\left(\delta_{i} / L\right)[F(k a)-G(k a)]$. The above quantity needs to be multiplied by a factor of 2 as we assume symmetric perturbation at $z= \pm a$.

We can draw certain conclusions from Eq. (26). By its very physical meaning, $S(k a)$ is positive as the energy released during the reconnection is thought to be responsible for the heating of the plasma and their subsequent acceleration. In order to plot function $S(k a)$, we note that close to the midnight meridian, the plasma sheet has a typical thickness of $\sim 6 R_{E}$ (Baumjohann and Paschmann, 1990), which remains unaffected during geomagnetic activity for the geocentric solar-magnetospheric distance $\mid X_{\mathrm{GSM}}<15 R_{E}$. We chose the magnetopause location at $\sim 30 R_{E}$ (Sibeck et al., 1986). Therefore, we chose $L \sim 6 R_{E}, a \sim 30 R_{E}$ with $\left|B_{z}\right| \sim 25 \mathrm{nT}$ and $\delta_{i} \sim 10^{4} \mathrm{~km}$. We plot $S(\mathrm{ka})$ against $\mathrm{ka}$ and see from Fig. 2 that for short scale length $k a \geq 5$, this function is positive. For $k a<5, S(k a)$ becomes negative.
This happens because tearing instability of the current sheet makes the perturbation approach applied here inappropriate. Even a small deformation can trigger a substantial reconfiguration of the initial magnetic field. Therefore, the above calculation is applicable only in the $k a \geq 5$ region.

To determine the instability threshold in the long wavelength limit $k a<5$, we rewrite Eq. (5) as a steady state Schrodinger equation (Treumann and Baumjohann, 1997)

$H A_{1}=E A_{1}$,

where Hamilton's operator $H=-d^{2} / d \bar{z}^{2}+2 / \cosh ^{2} \bar{z}$, and $A_{1}$ can be interpreted as the wave function localized in the interval $(-a, a)$ with a potential energy $V(z)$ equal to

$V(z)=-\frac{2}{\cosh ^{2}(z)}$,

and the energy eigenvalue $E=-\bar{k}^{2}<0$. A potential well $V(z)$ has to be deep enough to accommodate the localized state with a negative total energy of $E=-\bar{k}^{2}$. Thus, lower $E$ requires a deeper potential well. To determine the instability threshold of the tearing mode, we calculate the discontinuity in the logarithmic derivative of $A_{1}, \Delta$, defined below

$\Delta=\frac{1}{A_{1}(0)}\left[\left.\frac{d A_{1}}{d z}\right|_{z=+0}-\left.\frac{d A_{1}}{d z}\right|_{z=-0}\right]$,

with $\Delta>0$ corresponding to the tearing instability. This criteria yields

$\Delta=2 \frac{\left(1-\bar{k}^{2}\right)}{\bar{k}}$,

i.e. $\Delta>0$ implies $k a<a / L$. For parameters in Fig. 2, $a / L=5$, and we see that $k a<5$ coincides with the negative value of $S(k a)$. Physically it implies that when the equilibrium magnetic field is close to a magnetically stable state, as $k a$ approaches $a / L$ from above, $S(k a)$ becomes positive. When this happens, the tearing mode instability cannot occur.

Note that $\gamma<0$ implies that the driven reconnection will last for a short period $\tau \sim 1 /|\gamma|$ as within a few $\tau$, the reconnected flux saturates due to the balance between collisionless dissipation in the singular layer and the perturbed surface currents due to boundary distortion (Lakhina, 1992, 1996). Such short period reconnection may provide the required energy for BBFs. The spatial behaviour of the growth rate $\gamma$ shows that $\gamma<0$ for $k L \geq 1$ (curve 1, Fig. 2, Lakhina, 1992, 1996). This is consistent with the behaviour of $S(k a)$, with $k a$ as $k a \geq 5$ implies $k L \geq 1$ for $(a / L)=5$. Hence, it is clear that $S(k a)>0$ would imply a parametric regime where tearing modes are stable.

The implication on the energetics of reconnection is that the energy is provided by the changes in the topology of the magnetic field since topology controls the possible states of equilibrium of the plasma. Hence, even a small perturbation can trigger a significant relaxation in a marginally stable state. 


\section{Energy balance}

For typical parameters in ICPS (Baumjohann and Treumann, 1997) $n_{i} \sim 0.5 \mathrm{~cm}^{-3}, \rho_{H^{+}} \sim 5 \times 10^{-25} \mathrm{~g} \mathrm{~cm}^{-3}$, we estimate the kinetic energy density in a BBF per unit volume $\rho v^{2} / 2 \sim$ $10^{-9} \mathrm{erg} \mathrm{cm}^{-3}$ for $v \sim 600 \mathrm{~km} / \mathrm{s}$. A single reconnection event causes the $\Delta W$ release of energy dissipated over a time scale $\tau \sim 1 / \gamma$. Then, for a single reconnection event, for $B \sim 10^{-4}$ Gauss with $\delta \sim 0.3 R_{E}, a / L \sim 5$ and $L=$ $6 R_{E}$ and $S(\mathrm{ka}) \sim 1$ with $\delta_{i} \sim 10^{4} \mathrm{~km}$, we obtain $\Delta W \sim$ $10^{-9} \mathrm{erg} \mathrm{cm}^{-3}$. A part of the energy released due to a bursty driven reconnection goes into heating the plasma and the rest goes into acceleration. The energy going into acceleration is manifested as BBFs. Therefore, the energy released in a driven reconnection event is more than sufficient to explain the kinetic energy of BBFs.

Following Vekstein and Jain (1998), we estimate the power density emitted in an externally driven system. If external forcing time $T$ is short compared to the transition from $A^{i}$ to $A^{r}$ time $\tau \sim|\gamma|^{-1}$, a strong current sheet forms at the resonant surface $z=0$. Hence all of the energy difference, $\Delta W_{B}$, is available for plasma heating and subsequent acceleration. The heating rate is

$Q=\frac{\Delta W_{B}}{\tau} \sim|\gamma| \frac{B_{0}^{2}}{2 \mu_{0}}\left(\frac{\delta}{\delta_{i}}\right)^{2} S(k a) ; \quad T<\tau$.

If the external driving time is large compared to the transition time $\tau$, the transition to the reconnected state is almost complete and the current density inside the sheet is reduced by a $T / \tau$ factor. Then, the dissipation rate is

$Q=\frac{\Delta W_{B}}{\tau} \sim \frac{B_{0}^{2}}{2 \mu_{0}}\left(\frac{\delta}{\delta_{i}}\right)^{2}\left(\frac{\tau}{T^{2}}\right) S(k a) ; \quad \tau<T$.

The upper limit of dissipation is achieved at $\tau \sim T$, i.e.

$Q=\frac{\Delta W_{B}}{\tau} \sim \frac{B_{0}^{2}}{2 \mu_{0}}\left(\frac{\delta}{\delta_{i}}\right)^{2} T^{-1} S(k a) ; \quad \tau \sim T$.

The numerical simulation results of Birn et al. (1998) suggests that $T \sim(10-40) \tau_{A}$. Then $Q \sim 10^{-9} \mathrm{erg} \mathrm{cm}^{-3} \mathrm{sec}^{-1}$. This is the maximum power density in the inner plasma sheet region due to driven reconnection.

\section{Conclusions}

In the present work we have studied the energetic aspects of a driven reconnection model for bursty bulk flow. The total energy balance of a reconnection event suggests that redistribution in magnetic energy leads to the heating and acceleration of the plasma. The released magnetic energy during a reconnection event is comparable to the observed kinetic energy of BBFs, suggesting that the heating and acceleration of the ICPS particles is taking place simultaneously. In view of the above features, the driven reconnection model may provide a plausible mechanism for the generation of the BBFs.

Present analysis indicates that the driven reconnection is a possible candidate for triggering the BBFs. The driving force can be either pressure pulses (Russel et al., 1994) or quasi-steady fluctuations in the solar wind. However, observational constraints on the driving force and subsequently on the fluctuation amplitude as well as features like the local organisation of BBF events should be incorporated in a self-consistent model and the present model does not include these features. We shall address these issues in our future work.

The recent investigation on driven reconnection (Vekstein and Jain, 1999; Ishizawa and Tokuda, 2000) suggests that the typical relaxation type of plasma heating depends on the driving frequency. However, unlike solar coronal plasma, where a constant driver is required for the constant heating of the corona, in the bursty flows, which rise and fall over a period of a second, a sudden deformation of the boundary (i.e. a sudden change of pressure pulse) and the ensuing single reconnection event is sufficient. In this sense, the driven problem of solar coronal heating and bursty bulk flow are dissimilar.

Acknowledgement. Topical Editor G. Chanteur thanks V.S. Semenov and another referee for their help in evaluating this paper.

\section{References}

Angelopolous, V. et al., Bursty bulk flows in the inner central plasma sheet, J. Geophys. Res., 97, 4027, 1992.

Angelopolous, V. et al., Statistical characteristics of bursty bulk flows, J. Geophys. Res., 99, 21257, 1994.

Angelopolous, V. et al., Magnetotail flow bursts: association to global magnetospheric circulation, relationship to ionospheric activity and direct evidence for localization, Geophys. Res. Lett, 24, 2271, 1997.

Baumjohann, W. and Paschmann, G., The near-Earth plasma sheet, J. Geophys. Res., 95, 10707, 1990.

Baumjohann, W., The near-Earth plasma sheet: An AMPTE/IRM perspective, Space Sci. Rev., 64, 141, 1993.

Baumjohann, W. and Treumann, R. A. in Space Plasma Physics, Imperial College Press, London, 1997.

Birn, J., Computer studies of the dynamic evolution of the geomagnetic tail, J. Geophys. Res., 85, 1214, 1980.

Birn, J., Sommer, R. R., and Schindler, K., Open and closed magnetospheric tail configurations and their stability, Astrophys. Space Sci., 35, 389, 1975.

Birn, J., Hesse, M., and Schindler, K., Formation of thin current sheets in space plasmas, J. Geophys. Res., 103, 6843, 1998.

Browning, P. K. et al., Numerical study of nonlinear forced magnetic reconnection, Phys. Plasmas, 8, 132, 2001.

Hahm, T. S and Kulsrud, R. M., Forced magnetic reconnection, Phys. Fluids, 28, 2412, 1985.

Hones, E. W. Jr., The magnetotail: Its generation and dissipation in physics of solar planetary environments, Ed. D. J. Williams, p. 558, American Geophysical Union, Washington DC, 1976.

Hones, E. W. Jr. and Schindler, K., Magnetotail plasma flow during substorms, a survey with IMP6 and IMP8, J. Geophys. Res., 84, $7155,1979$.

Ishizawa, A. and Tokuda, S., Improved boundary layer analysis of forced magnetic reconnection due to boundary perturbations, Phys. Plasmas, 7, 875, 2000. 
Lakhina, G. S. and Schindler, K., The effect of plasma-sheet boundary flow and plasma mantle flow on the ion tearing instabilities, J. Geophys. Res., 93, 8591, 1988.

Lakhina, G. S., A kinetic theory of driven reconnection in the Earth's Magnetotail, J. Geophys. Res., 97, 2961, 1992.

Lakhina, G. S., A mechanism for bursty bulk flows in the plasma sheet region, in Third international conference on substorms (ICS-3, Versailles, France, 12-17 May 1996), ESA SP-389, 2330, 1996.

Russel, C. T. et al., Effects of sudden impulses on currents in the auroral ionosphere under northward IMF conditions: A case study, J. Geophys. Res., 97, 17617, 1994.

Schindler, K., A self consistent theory of the tail of magnetosphere, in Earth's Magnetospheric processes, Ed. B. M. McCormac, p. 200, D. Riedel, Hingham, Mass., 1972.
Sibeck et al., Major flattening of the distant geomagnetic tail, J. Geophys. Res., 91, 4223, 1986.

Sergeev, A. V. et al., In situ observations of magnetotail reconnection prior to the onset of small substorm, J. Geophys. Res., 100, 19121, 1995.

Treumann, R. A. and Baumjohann, W., in Advanced Space Plasma Physics, Imperial College Press, London, p. 163, 1997.

Vekstein, G. E. and Jain, R., Energy release and plasma heating by forced magnetic reconnection, Phys. Plasmas, 5, 1506, 1998.

Vekstein, G. E. and Jain, R., On plasma heating by reconnective magnetic relaxations, Phys. Plasmas, 6, 2897, 1999.

Yermolaev, Yu. I. et al., Two spacecraft observation of plasma sheet convection jet during continuous external driving, Geophys. Res. Lett., 26, 177, 1999. 